\title{
Scale-invariant avalanche dynamics in the temperature-driven martensitic transition of a Cu-Al-Be single crystal
}

\author{
Francisco Javier Romero, José-María Martín-Olalla, and María Carmen Gallardo \\ Departamento de Física de la Materia Condensada. Universidad de Sevilla. P.O. Box 1065, E-41080 Sevilla, Spain \\ Daniel Soto-Parra \\ Instituto Potosino de Investigación Científica y Tecnológica. A. C., Camino de la Presa San José 2055, \\ Col. Lomas 4a, CP 78216 San Luís Potosí, S. L. P. \\ and Tecnológico Nacional de México/Instituto Tecnológico de Delicias, Paseo Tecnológico km. 3.5, Cd. Delicias, Chihuahua 33000, Mexico
}

Ekhard K. H. Salje

Department of Earth Sciences. University of Cambridge. Dawning Street, Cambridge CB2 3EQ, United Kingdom

Eduard Vives and Antoni Planes

Departament de Física de la Matèria Condensada, Facultat de Física. Universitat de Barcelona, Diagonal, 647, E-08028 Barcelona, Catalonia

(Received 23 March 2019; published 20 June 2019)

\begin{abstract}
We have combined high sensitivity, extra-low differential temperature scanning rate calorimetry, and acoustic emission (AE) measurements to study avalanches during the cubic $\leftrightarrow 18 \mathrm{R}$ martensitic transition of a Cu-Al-Be single crystalline shape memory alloy. Both AE and calorimetry corroborate a good power-law behavior for cooling with an exponent $\varepsilon \simeq 1$.6. For heating, a slope is observed in the maximum likelihood curves, which confirms that our data are affected by an exponential cutoff. An effective energy exponent, $\varepsilon \sim 1.85$, and a cutoff, $\lambda^{-1}=0.115(38) \times 10^{-3} \mathrm{aJ}$, were determined by fits of power-laws with exponential damping. The long tail observed in the low-temperature region by calorimetric measurements suggests the existence of significant elastic effects that constrain the progress of the transformation at low temperatures. While thermodynamic features such as transformation enthalpy and entropy are those expected for $\mathrm{Cu}$-based shape-memory alloys undergoing a cubic $\leftrightarrow 18 \mathrm{R}$ transition, the critical behavior deviates from the corresponding behavior expected from this symmetry change. These deviations are a consequence of the elastic hardening induced by the interplay of the transformation with dislocation jamming, which has the effect of effectively reducing the number of pathways connecting the parent and martensitic phase.
\end{abstract}

DOI: 10.1103/PhysRevB.99.224101

\section{INTRODUCTION}

Martensitic transitions transform a high-symmetry crystal structure (parent phase) to a lower-symmetry structure (martensite) through a diffusionless, shear-dominant mechanism $[1,2]$. They are well-known examples of physical processes that occur intermittently through successive avalanches [3-5]. This avalanche dynamics is due to the existence of kinetic impediments, such as disorder or compatibility constraints that configure a complex energy landscape with many metastable states that characterize the region of coexistence of the high- and low-symmetry phases. The transition occurs through successive strain relaxations that connect these metastable states and avalanches are associated with these fast relaxation processes. In most martensitic materials this behavior is favored by the fact that thermal fluctuations are irrelevant so that the dynamics in these systems is athermal to a good approximation. This is a consequence of the highenergy barriers separating metastable states in the coexistence region. Therefore, the transformation process only occurs when the system is externally driven, which entails that the transition extends over a finite temperature interval. The study of avalanches in martensitic transitions has raised a lot of interest since it provides relevant information about the collective behavior of nonequilibrium transformation processes, which is expected to take into account both the individual growth/shrinkage of martensitic domains and their interaction, which is dominated by long range elastic effects.

Avalanches can be detected by means of many different experimental techniques. The essential requirement is that the technique must be highly sensitive to small local changes of the internal strain field, and must have a very good time resolution. The observation of acoustic emission (AE), which is based on the detection of the high frequency transitory acoustic waves associated with changes of the internal strain field, is acknowledged to be the most adequate experimental method to study avalanches during martensitic transitions [3]. However, other techniques can provide complementary information about avalanche dynamics. In particular, the use of high sensitivity differential scanning calorimetry provide an interesting alternative, which enable us to determine the absolute value of the energy associated with avalanches [6]. 
In martensitic transitions, avalanches often take place under the absence of characteristic scales. This scale-free dynamics, usually known as avalanche criticality, is reflected by power-law distributions of the energy and duration of the avalanches [3-5,7]. It has been shown that critical exponents can be grouped into classes that depend to a large extent on the symmetry reduction that occur at the martensitic phase. More specifically, the exponents are determined by the ratio between symmetry operations in the parent and martensitic phases that determines the degeneracy of the low-temperature phase or variant multiplicity (number of energetically equivalent domains). The idea behind this fact is that by increasing variant multiplicity, the system is able to find more pathways connecting high and low symmetry phases thus making the transition more adaptive. Then, the probability of large events should decrease compared with the probability of small ones, which should result in size, energy, and duration distributions with larger critical exponents.

In the present paper we have used a bespoke designed high sensitivity calorimeter that can work at extra-low-temperature rates, close to the limit where thermal activated effects are expected to play a role, to study avalanches during the martensitic transition of a $\mathrm{Cu}-\mathrm{Al}-\mathrm{Be}$ single crystal. These measurements have been used to complement the $\mathrm{AE}$ results. $\mathrm{Cu}-\mathrm{Al}-\mathrm{Be}$ belongs to the $\mathrm{Cu}$-based family of shape-memory alloys. These materials show a cubic phase at high temperature (above $1000 \mathrm{~K}$ ) that can be retained at room temperature by means of fast enough cooling. Usually, during cooling the sample transforms first to an ordered $\mathrm{DO}_{3}(\mathrm{Fm} 3 \mathrm{~m})$ structure and martensitically transforms towards a lower symmetry phase at a lower temperature.

We have focused on $\mathrm{Cu}-\mathrm{Al}-\mathrm{Be}$ shape memory alloys because these shape-memory materials show unusual mechanical behavior related to the martensitic transition. Only parentmartensite interfaces are somewhat mobile in $\mathrm{Cu}-\mathrm{Al}-\mathrm{Be}$, while interfaces between martensitic variants are immobile. The pinning of these interfaces has been shown in Ref. [8] to stem from a complex interplay between the martensitic transformation and dislocations. Dislocations in the martensitic phase are partially inherited from the parent phase and partially created by the transformation. The later are basal plane dislocations that are responsible for a dislocation jamming effect of the growing interfaces, and thus dominate the microstructure that is formed during the transition. This jamming mechanism is expected to affect avalanche criticality by effectively reducing the number of pathways connecting the parent and martensitic phase, which is expected to affect the critical exponents of avalanche dynamics. This paper describes a detailed study of avalanche behavior in $\mathrm{Cu}-\mathrm{Al}-\mathrm{Be}$ single crystals and analyzes the influence of dislocation jamming on avalanche criticality. The paper is organized as follows. Experimental details are given in Sec. II. AE and calorimetric results are presented and discussed in Sec. III. Finally, a brief summary of our work is provided in Sec. IV.

\section{EXPERIMENTAL}

A $\mathrm{Cu}-\mathrm{Al}-\mathrm{Be}$ single crystal was prepared by a modified Bridgman method. The atomic composition is $\mathrm{Cu}_{74.08}$ $\mathrm{Al}_{23.13} \mathrm{Be}_{2.79}$ and was determined by microprobe analysis of
$\mathrm{Cu}$ and $\mathrm{Al}$ while the Be content was inferred from the composition of the melt. The specimen used for $\mathrm{AE}$ and calorimetric measurements was cut with a low-speed diamond saw from the same rod from which the sample studied in Ref. [8] was obtained. The present sample is nearly rectangular with flat faces [parallel to the (110) plane of the cubic phase] of area $70.6 \mathrm{~mm}^{2}$ and $0.5 \mathrm{~mm}$ height. The mass of the sample is 0.199 g. Previous to the experiments, the specimen was heat treated at $\sim 1100 \mathrm{~K}$, cooled in air down to room temperature, and aged at room temperature for a time long enough (about three months) to ensure that the sample reached a highly ordered final state, free from internal stresses and with a minimum vacancy concentration. Next, the sample was cycled several times across the transition to avoid the influence of initial cycling effects on the transition, which is known to occur during the first transformation runs in virgin samples [9]. The parent phase is cubic $(F m 3 m)$, and the martensitic phase has a monoclinic long-period structure which is usually denoted as 18R [10].

For AE measurements, the sample was mounted on top of a copper block situated inside a double Faraday cage constituted of cooper and iron shielding layers. It was heated and cooled by circulating a temperature-controlled fluid, which allows to establish linear temperature ramps with selected rates in the range from $10^{-3} \mathrm{~K} / \mathrm{s}$ to $10^{-1} \mathrm{~K} / \mathrm{s}$. The $\mathrm{AE}$ was detected with a piezoelectric transducer (micro- 80 transducer, encapsulated in stainless steel) acoustically coupled to the upper surface of the sample by a thin layer of petroleum jelly. The output voltage of the transducer was preamplified by $40 \mathrm{~dB}$, band filtered between 100 and $400 \mathrm{kHz}$ and transferred to a PCI2 digital acquisition system (Europhysical Acoustics). Data were stored at a sampling rate of $10 \mathrm{MHz}$.

For identification of AE events associated with avalanches, a threshold above the instrument noise was fixed. An event is assumed to start when the signal crosses for the first time a voltage threshold at a time $t_{\mathrm{i}}$. The event will end at $t_{f}=$ $t_{i}+\Delta t_{i}$ corresponding to the time at which the voltage crosses the threshold in the downwards direction and remain below the threshold for more than a preselected time (or hit detection time, HDT) that was set to $100 \mu \mathrm{s}$ in our experiments. The amplitude of an AE event is defined as the maximum of the corresponding voltage, and its energy can be estimated by numerical integration of the square voltage during the duration $\Delta t_{i}$, normalized by a reference electric resistance, $R(=10 \mathrm{k} \Omega$, in our case).

The AE activity is defined as the cumulated number of events per time or temperature interval. The interval is taken long enough (of the order of seconds) so that this quantity can be compared with calorimetric measurements.

Measurements of heat flux $\Phi(=d Q / d t)$ were performed using a high-resolution conduction calorimeter, which has been described elsewhere [11]. The sample is pressed between two identical heat fluxmeters, which are made from 50 chromel-constantan thermocouples connected in series, with the wires placed in parallel lines. The assembly is placed in a cylindrical hole axially located in a bronze cylinder $(10 \mathrm{~kg})$ which constitutes the calorimeter block. The temperature of the block is measured with a commercial platinum thermometer (Leads and Northup model 8164B). The block and the two surrounding radiation shields are placed into a hermetic outer 


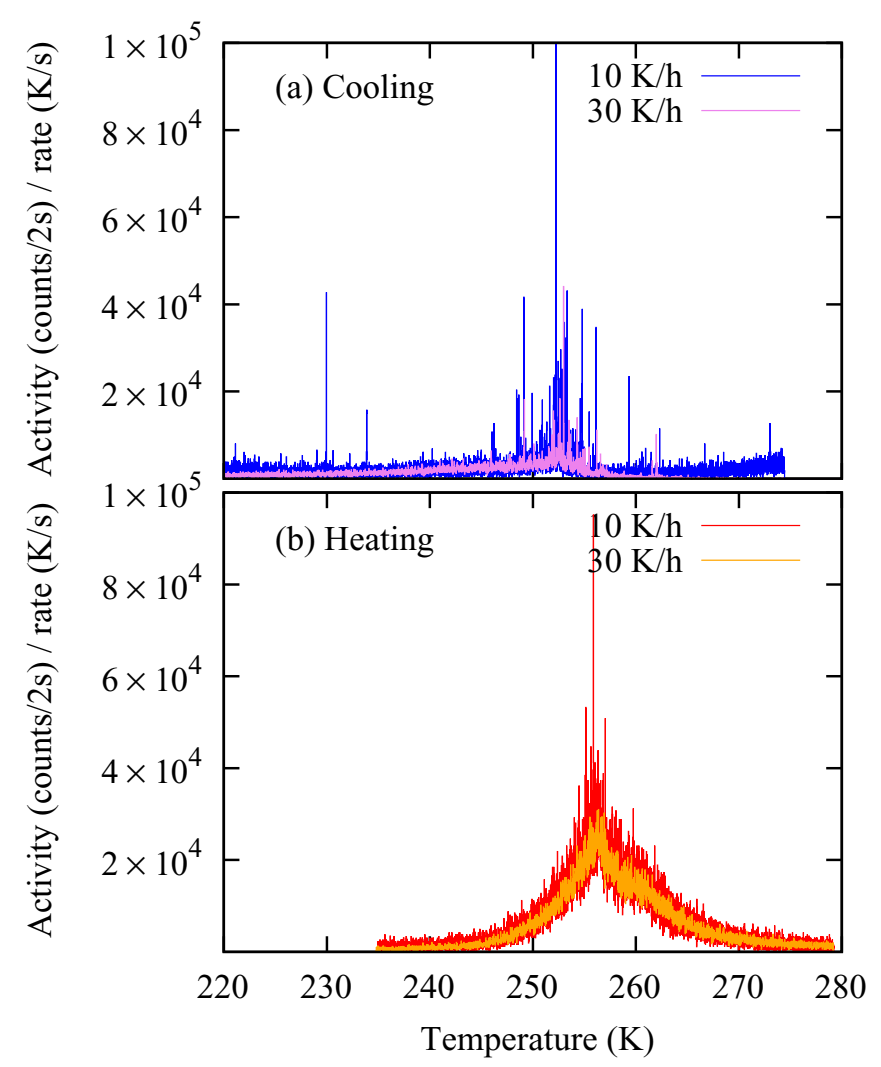

FIG. 1. Scaled representation of the AE activity for cooling (a) and heating (b) runs.

case under vacuum $\left(10^{-7}\right.$ torr). This assembly is surrounded by a coiled tube and placed in a Dewar jar filled with alcohol whose temperature is regulated by circulation of liquid $\mathrm{N}_{2}$ or Thermal H5 fluid.

The heat flux is evacuated through the fluxmeters, which allows the measurement of heat flux with a resolution better than $0.1 \mu \mathrm{W}$. Due to the high thermal inertia of the calorimeter block, very-low-temperature rates of some $10^{-7} \mathrm{~K} / \mathrm{s}$ (about $10^{-3} \mathrm{~K} / \mathrm{h}$ ) can reliably be controlled with temperature fluctuations of the calorimeter block lower than $10^{-6} \mathrm{~K}$. During cooling and heating runs, the system works as a differential thermal analysis device. The electromotive force provided by the fluxmeters, which is proportional to the heat flux, was recorded by a nanovoltmeter (Keithley K2182) at a sampling rate of $12.5 \mathrm{~Hz}$. The integral of the heat flux above a suitable baseline, $\Delta \Phi$, is proportional to the excess enthalpy associated with the transition [12].

\section{RESULTS}

\section{A. AE results}

Figure 1 shows the AE activity cumulated per unit of temperature, $v$, during cooling (a) and heating (b) runs as a function of temperature for two studied temperature rates. It is obtained as the cumulated activity measure in time intervals of $2 \mathrm{~s}$ divided by the local temperature rate, $r=\dot{T}(t)$. In this representation it is expected that when thermal activation effects are not relevant (athermal regime), $v$ becomes independent of the rate. The $v$ versus $T$ curves obtained at

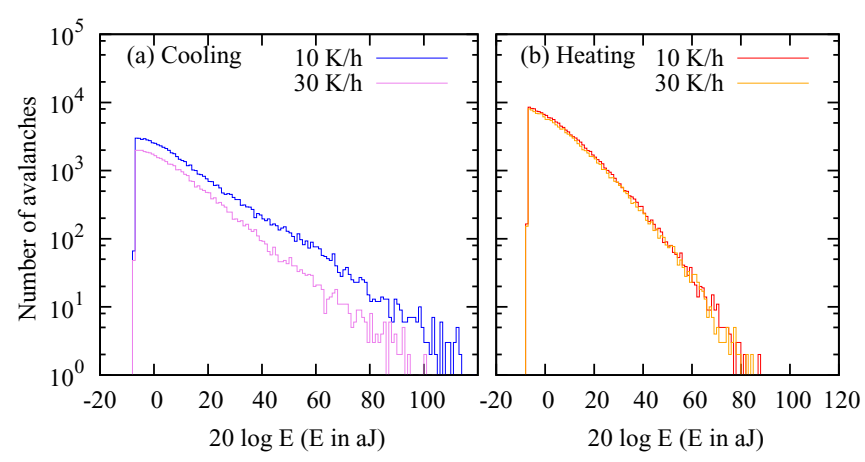

FIG. 2. Histograms representing the distribution of energies for the forward (a) and reverse (b) transitions corresponding to selected values of the temperature rate.

different rates should collapse into a single curve. Therefore, deviations from this behavior are ascribed to the occurrence of thermal activation effects. Indeed, it is expected that these activation effects become more and more relevant as the rate is decreased. Our AE results show a very good scaling in the range from $10 \mathrm{~K} / \mathrm{h}$ to $30 \mathrm{~K} / \mathrm{h}\left(\sim 2.5 \times 10^{-3} \mathrm{~K} / \mathrm{s}\right.$ to $\left.10^{-2} \mathrm{~K} / \mathrm{s}\right)$, which indicates that thermal activation effects are irrelevant in this rate regime. Note that the larger scatter of the curves corresponding to lower rates is simply due to the propagation of the error in the temperature measurements.

The probability distribution functions of $\mathrm{AE}$ energies are shown in Fig. 2. The corresponding histograms are presented in a log-log scale and suggest an excellent power law behavior for cooling data characterized by a well-defined exponent over six decades (20 dB represents one decade). For heating, some deviation from power law are clearly observed, which suggests that the data are affected by an exponential damping. To confirm the behaviors foreseen for cooling and heating we have numerically studied the dependence of the exponent $\varepsilon$ fitted using the maximum likelihood method as a function of a varying lower cutoff $E_{\min }$. This analysis should lead to a significant plateau that defines the exponent in the case of a power law distribution [13]. However, when results are affected by an exponential damping, the maximum likelihood analysis should reveal, instead of the plateau, a positive slope of the fitted exponent versus the cutoff $E_{\min }$ [14]. The maximum likelihood analysis corresponding to the data presented in Fig. 2 is shown in Fig. 3. Results confirm a good power law behavior for cooling with an exponent $\varepsilon=1.6 \pm 0.1$. For heating, a clear slope is observed, which confirms the existence of damping. An effective energy exponent, $\varepsilon \sim$ 1.85 , and a damping cutoff, $\lambda^{-1}=0.115(38) \times 10^{-3}$ aJ, were estimated by comparing experimental results to synthetic data that reproduce a biparametric probability density function of the form $\propto E^{-\varepsilon} e^{-\lambda E}$. It is worth mentioning that the different behavior of the avalanches in the forward and reverse transitions is in good agreement with results reported for $\mathrm{Cu}-\mathrm{Zn}-\mathrm{Al}$ shape-memory alloy in Ref. [15], which undergo a martensitic transition with the same change of symmetry.

\section{B. Calorimetric results}

The temperature dependence of the heat flux $\Phi$ was recorded at the following rates: $r=-0.04 \mathrm{~K} / \mathrm{h}, r=-0.25$ 

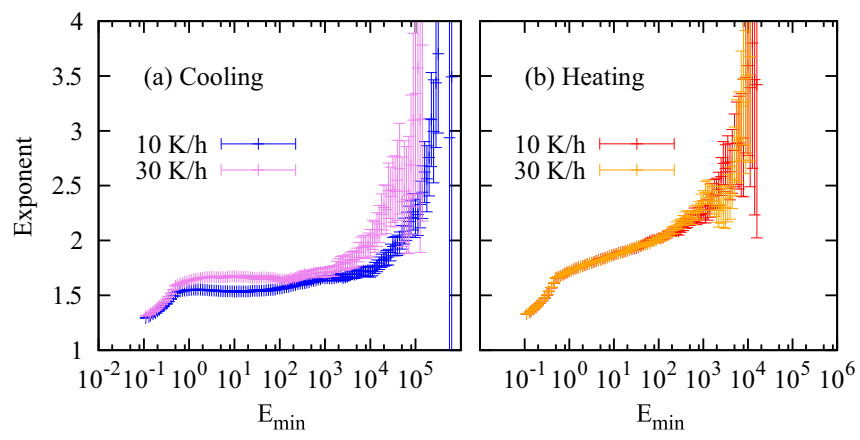

FIG. 3. Fitted exponent as function of the cutoff $E_{\min }$ for $\mathrm{AE}$ experiments for cooling (a) and heating (b) runs at selected temperature rates. Cooling runs yield power law behavior with an exponent close to 1.6. Heating data (b) are clearly affected by an exponential damping.

$\mathrm{K} / \mathrm{h}$, and $r=-0.40 \mathrm{~K} / \mathrm{h}$ for cooling runs and $r=0.04 \mathrm{~K} / \mathrm{h}$, $r=0.20 \mathrm{~K} / \mathrm{h}$, and $r=0.50 \mathrm{~K} / \mathrm{h}$ for heating runs. Although individual jerks are visible in both cooling and heating runs, the jerky character of the thermal signal is more prominent for the forward transformation than for the reverse one, as clearly indicated by the occurrence of more energetic spikes during cooling (see Fig. 4). The heat flux scaled by the temperature rate (normalized heat flux $\Phi / r$ ) gives the amount of released energy per unit temperature. The excess of normalized heat flux relative to an appropriate baseline is shown in Fig. 5 for cooling rates $r=-0.40 \mathrm{~K} / \mathrm{h}$ and $r=-0.25 \mathrm{~K} / \mathrm{h}$ and heating rates $r=+0.50 \mathrm{~K} / \mathrm{h}$ and $r=+0.20 \mathrm{~K} / \mathrm{h}$. Scaling of the thermal curves is again observed to a quite good approximation. This result confirms that athermal behavior is satisfied for rates above $0.25 \mathrm{~K} / \mathrm{h}$. It is worth noting that in both cooling and heating curves a broad and smooth heat flux bump is observed, which is expected to be constituted from a large number of superimposed spikes.

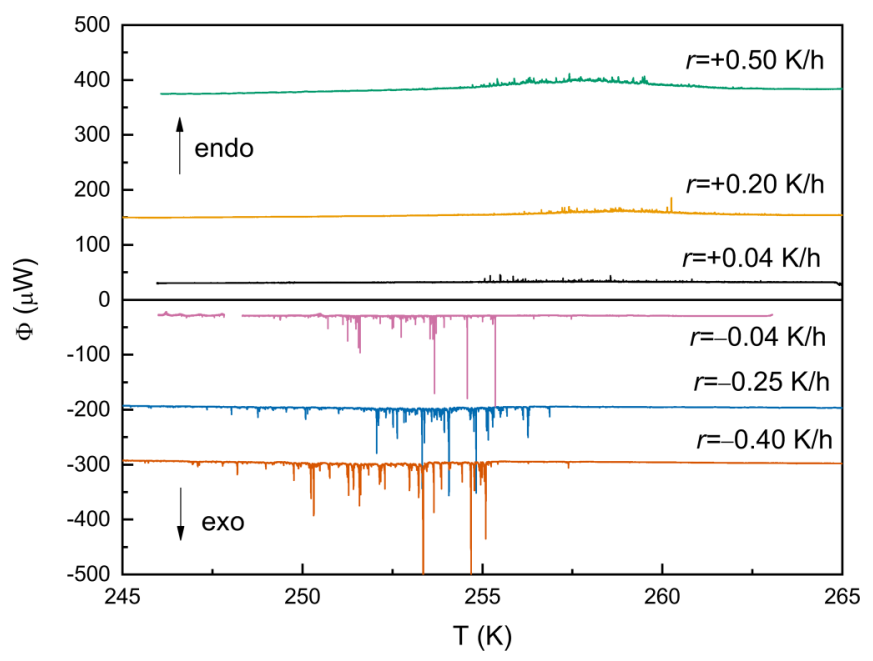

FIG. 4. Heat flux $\Phi$ as a function of temperature for heating (top, endothermic curves) and cooling (bottom, exothermic curves) runs at several temperature rates. A series of spikes is evident on both the reverse and forward transformations indicating the jerky behavior of the transition.

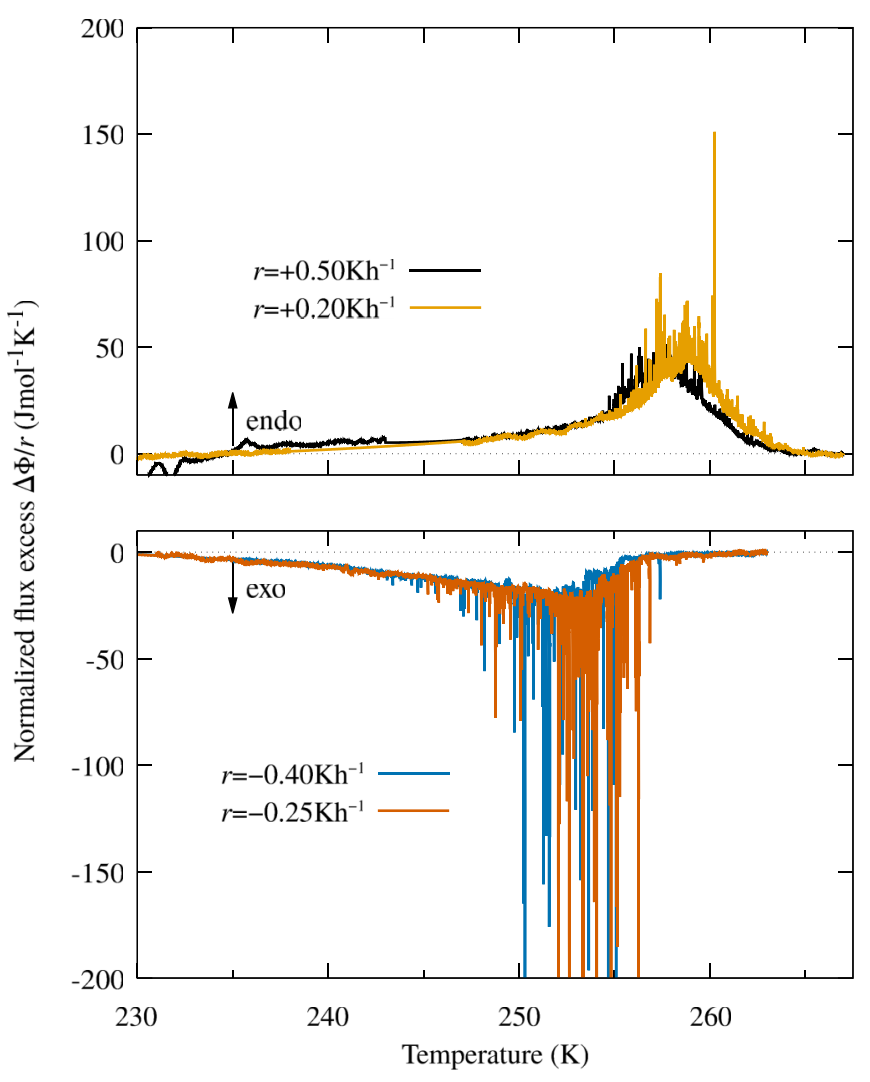

FIG. 5. Normalized heat flux $\Delta \Phi / r$ as a function of temperature for heating (top, endothermic) and cooling (bottom, exothermic) at two temperature rates. The area subtended by each run above the base line (dotted lines) equals the excess enthalpy.

The integral over temperature of $\Delta \Phi / r$ gives the excess enthalpy of the transformation. This is shown in Fig. 6. The long tails observed in the low-temperature region are worth noting and suggest the existence of significant elastic

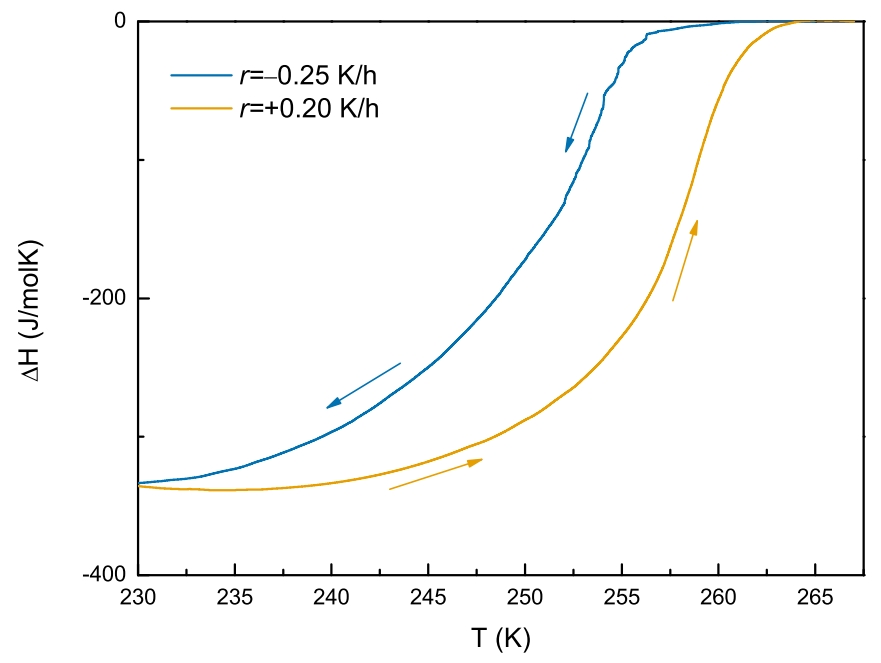

FIG. 6. Excess enthalpy computed by integrating of the normalized heat flux for data shown in Fig. 5. Hysteresis is evident with both curves displaced $\Delta T=8 \mathrm{~K}$ and different rounding mechanisms. 


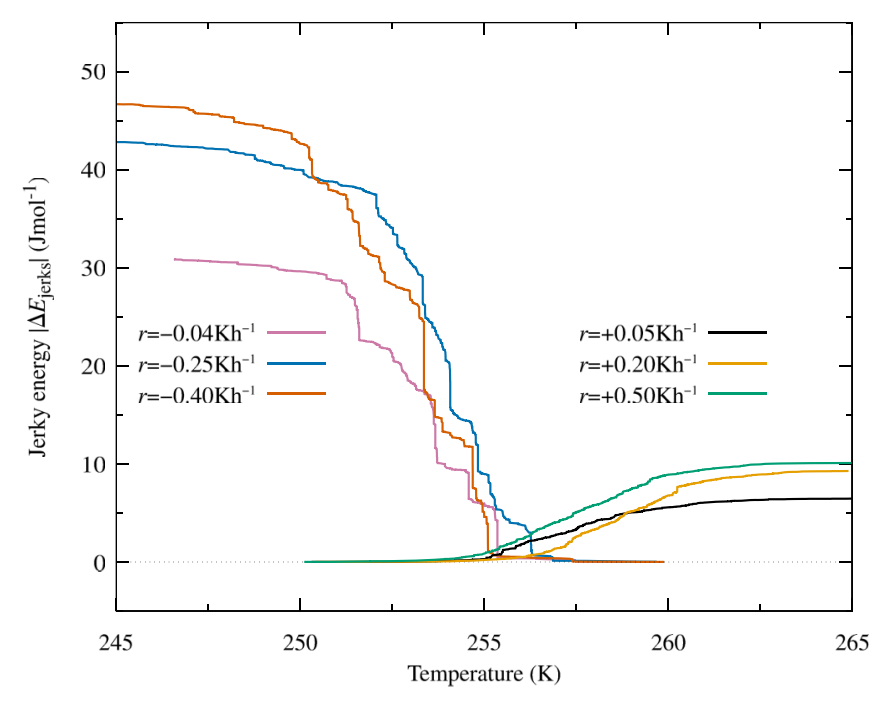

FIG. 7. Cumulative thermal avalanche energy for cooling and heating runs, at selected scanning rates. Monotonous increasing avalanche energy with increasing the rate is observed both for the forward and reverse transitions. Total avalanche energy, $E_{\text {th }}$, is five times larger in the forward than in the reverse transition. This difference is also apparent in Figs. 4 and 5.

effects that constrain the progress of the transformation at low temperatures.

Within experimental uncertainties, the whole excess enthalpy corresponds to the latent heat of the transition. Similar values were found for all runs, showing that the excess enthalpy is indeed independent of the rate of the temperature change. The average value is $\Delta H_{t}=340(10) \mathrm{J} / \mathrm{mol}$, corresponding to all heating and cooling runs. This value is in good agreement with the latent heat reported previously for a sample with a similar composition [16,17]. The entropy excess is estimated as $\Delta S \simeq \Delta H_{t} / T_{0}$, where $T_{0}$, defined as the average temperature between heating and cooling loops, is $255 \mathrm{~K}$, which yields $\Delta S=1.33(4) \mathrm{J} / \mathrm{mol} \mathrm{K}$, in agreement with previous calorimetric estimates for $\mathrm{Cu}$-based shapememory alloys transforming to a $18 \mathrm{R}$ martensitic phase [18].

With the aim of analyzing the distribution of the energy of the spikes, which are assumed to be related to big avalanches, the calorimetric signal was filtered by a fifth-order all-pole Butterworth filter with a normalized cut-off frequency of $8 \times 10^{-2} \mathrm{~Hz}$. Searching for local maxima in the filtered signal allowed to characterize spike occurrence by position and height [19]. Peak height can then be converted into avalanche energy $E$ by multiplying by the characteristic relaxation time $\tau$ of the calorimetric setup. The relaxation time $\tau=65 \mathrm{~s}$ was estimated from the exponential decay in the fluxmeter signal following a quasi-instantaneous heat pulse.

The catalogue of avalanche/jerky events can be analyzed in different ways. First the cumulative energy associated with these avalanches can be computed as a function of temperature. Figure 7 shows that the avalanche energy content is very different for cooling and heating runs. In both regimes this energy shows a significant dependence on the scanning rate. During the forward transformation (cooling) the avalanche energy is about five time greater than the corresponding energy of the reverse transformation, which confirms the asymmetry in the dynamics of both transformations. The avalanche energy in the forward transformation amounts to about $15 \%$ of the total enthalpy, while it represents only about $3 \%$ of the whole enthalpy in the reverse transformation. This result confirms that strain energy relaxation is significantly different in the forward and reverse transition as suggested in Ref. [20]. In fact, if thermoelastic equilibrium was strictly satisfied, then strain energy relaxation should only occur in the forward transition due to nucleation while the reverse transition should take place by shrinkage of the martensitic variants with no additional energy loss.

A monotonous increase of the avalanche energy with the scanning rate is clearly suggested by data given in Fig. 7 in cooling and heating runs. The effect is especially remarkable in the case of cooling where the avalanche energy increases by more than $30 \%$ when the rate increases from $0.04 \mathrm{~K} / \mathrm{h}$ to $0.4 \mathrm{~K} / \mathrm{h}$.

It is worth noting that the number of detected thermal avalanches is much lower than the corresponding number of AE avalanches (see Table I). Nevertheless, avalanches detected in calorimetry have energies orders of magnitude larger than AE avalanches. This not only suggests that calorimetric avalanches should be understood as a sum of small AE avalanches that occur strongly correlated in time but also that

TABLE I. Number of detected calorimetric events and cumulative jerky energy for different runs.

\begin{tabular}{lrrr}
\hline \hline$r(\mathrm{~K} / \mathrm{h})$ & No. thermal avalanches & $E_{\mathrm{th}}(\mathrm{J} / \mathrm{mol})$ & No. AE avalanches \\
\hline Cooling & & & \\
-0.04 & 829 & 30.9 & \\
-0.25 & 1235 & 43.9 & \\
-0.40 & 879 & 47.9 & 62504 \\
-10 & & & 39298 \\
-30 & & & \\
Heating & & & \\
0.04 & 543 & 6.5 & \\
0.25 & 818 & 9.3 & \\
0.40 & 852 & 10.2 & 10.4 \\
10 & & & 146701 \\
30 & & & 134529 \\
\hline \hline
\end{tabular}




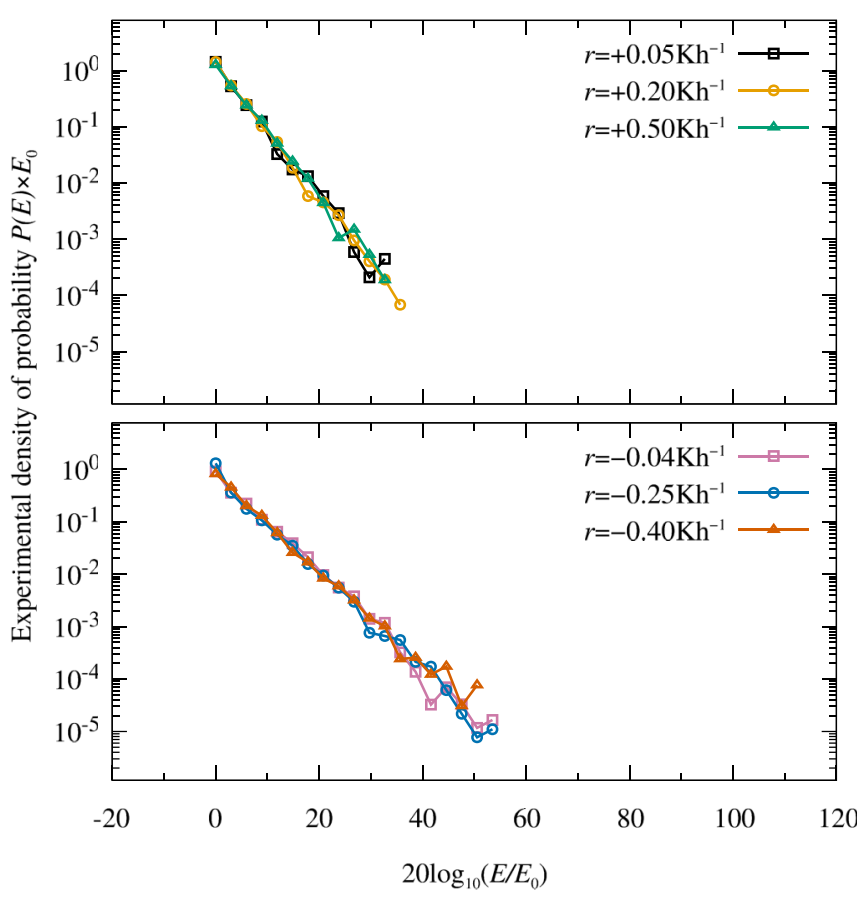

FIG. 8. Histograms representing the distribution of thermal avalanche energies for the reverse (top) and forward (bottom) transformations at several rates. Vertical axis displays counts per unit bin length scaled by the minimum observed energy $E_{0}=14 \mu \mathrm{J}$. Data were distributed in $N=20$ bins logarithmically spaced from $E_{0}$ to the highest event. For the sake of comparison axes kept the ranges displayed in Fig. 2.

the continuous background displayed by the thermal curves (broad heat flux bump) is, in fact, largely constituted of a superposition of uncorrelated very small avalanches. Note that the increase of the avalanche energy with the rate is consistent with this point of view as by increasing the rate, the overlap effect increases, and consequently the energy content of the thermal spikes increases too.

From Table I, it is worth noting that the number of $\mathrm{AE}$ avalanches is much larger in the reverse than in the forward transition. At a first glance this might seem paradoxical since the forward transition has a more prominent jerky character than the reverse transition. We suggest that this behavior is due to the existence of a very large number of tiny (but still detectable) AE avalanches during the reverse transition that remain hidden in the continuous calorimetric background. Actually, the existence of this large number of small avalanches is consistent with the lack of avalanche criticality during the reverse transition.

The distribution of thermal avalanche energies is depicted in Fig. 8. It is worth noting that this distribution of energies is not influenced by the temperature rate. Figure 9 shows the corresponding maximum likelihood plots for cooling and heating runs. For cooling an exponent $\varepsilon=1.7 \pm 0.1$ is found, which is compatible with the exponent obtained from $\mathrm{AE}$ measurements. For heating a larger exponent of $\sim 2.5$ is observed. This large value might be due to poor statistics for the heating runs. Nevertheless, if one assumes that data are affected by an exponential cutoff, a biparametric probability

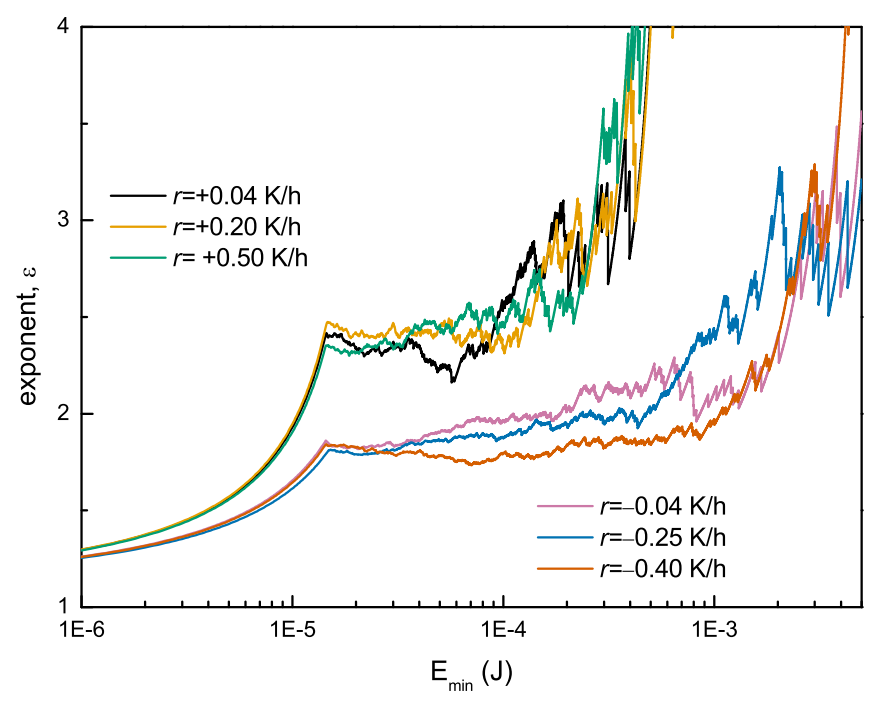

FIG. 9. Maximum likelihood plots giving the fitted exponent as function of the cutoff energy for calorimetric experiments.

density function of the form $\propto E^{-\varepsilon} e^{-\lambda E}$ can be used to fit the data. The fit renders an effective exponent of 1.86 in very good agreement with the effective exponent obtained with a similar biparametric fit of the AE data.

\section{SUMMARY}

We have studied avalanches during the martensitic transition of a $\mathrm{Cu}-\mathrm{Al}-\mathrm{Be}$ single crystal by means of $\mathrm{AE}$ and calorimetric measurements. To a certain extent, this material behaves similar to other $\mathrm{Cu}$-based shape memory materials. In particular, for temperature rates larger than $5 \times 10^{-5} \mathrm{~K} / \mathrm{s}$, the dynamics is clearly athermal and displays avalanche criticality to a very good approximation during the forward transition. However, deviations from critical behavior have been observed during the reverse transition. This asymmetry between heating and cooling is detected both in calorimetry and AE measurements. Our results are in agreement with previous data reported in Ref. [15]. The asymmetry has been explained [15] by the fact that while nucleation is required for the forward transition from the parent to martensitic phase, the reverse transition occurs by fast shrinkage of martensitic domains. This dynamic asymmetry influences the distribution of avalanches, which is critical in the forward transitions but exponentially damped in the reverse transition. Similar results have been obtained in mechanically-induced transitions [21] and in magnetic shape memory materials [20]. For this class of materials, the authors of Ref. [20] suggested that the asymmetry is associated with different mechanisms of the relaxation of elastic strain energy in the forward and reverse transitions, which might also explain the fact that the nature of the asymmetry can be different in different materials. This point of view complements the interpretation based on nucleation and in fact consistent with the different cumulated jerky energies calorimetrically measured during cooling and heating in the present work, which represents a good estimate of the dissipated energies in the corresponding forward and reverse transitions (see Fig. 7). 
Critical behavior during the forward transition is confirmed both from AE and calorimetric measurements. This is interesting since these two experimental techniques detect avalanches at very different scales (low energies in the case of $\mathrm{AE}$, and high energies in calorimetry). This result supports the idea of scale invariance. Nevertheless, the exponents obtained from both techniques are not identical as the calorimetric exponent is slightly higher than the AE exponent. This may be simply a consequence of quite poor statistics in this kind of avalanche measurements, however.

The obtained critical exponent associated with the distribution of $\mathrm{AE}$ energies, $\varepsilon \simeq 1.6$, is smaller than the expected exponent for systems undergoing a cubic to monoclinic transition. For this change of symmetry 12 energetically equivalent domains can grow at the transition in the absence of externally applied stress. For this variant multiplicity the expected critical energy exponent is $\varepsilon \simeq 2$ [4]. The lower value obtained here must be understood taking into account that in the studied materials the martensite phase is elastically much harder than the parent high temperature phase, which is a consequence of pinning effects arising from dislocation jamming. This is an unusual behavior that suggests that the growth of some variants may be inhibited during the transformation. Consequently this may lead to an effective reduction of variant multiplicity, which give rise to the lower critical exponent confirmed in the present work. The existence of pinning effect is also consistent with the log tails of the transformation in the low-temperature region as detected from calorimetric measurements (see Fig. 6).

\section{ACKNOWLEDGMENTS}

We acknowledge financial support from CICyT (Spain) (Project No. MAT2016-75823-R). E.K.H.S. thanks the EPSRC for support (Grant No. EP/P024904/1).
[1] M. Ahlers, The martensitic transformation: Mechanisms and crystallography, Philos. Mag. A 82, 1093 (2002).

[2] K. Battacharya, Microstructure of Martensite (Oxford University Press, Oxford, 2003).

[3] E. Vives, J. Ortín, L. Mañosa, I. Ràfols, R. Pérez-Magrané, and A. Planes, Distributions of Avalanches in Martensitic Transformations, Phys. Rev. Lett. 72, 1694 (1994).

[4] A. Planes, L. Mañosa, and E. Vives, Acoustic emission in martensitic transformations, J. Alloys Compd. 577, S699 (2013).

[5] E. K. H. Salje and K. A. Dahmen, Crackling noise in disordered materials, Annu. Rev. Condens. Matter Phys. 5, 233 (2014).

[6] M. C. Gallardo, J. Manchado, F. J. Romero, J. del Cerro, E. K. H. Salje, A. Planes, E. Vives, R. Romero, and M. Stipcich, Avalanche criticality in the martensitic transition of $\mathrm{Cu}_{67.64} \mathrm{Zn}_{16.71} \mathrm{~A}_{15.65}$ shape-memory alloy: A calorimetric and acoustic emission study, Phys. Rev. B 81, 174102 (2010).

[7] D. L. Beke, L. Daróczi, L. Z. Tóth, M. K. Bolgár, N. M. Samy, and A. Hudák, Acoustic emissions during structural changes in shape memory alloys, Metals 9, 58 (2019).

[8] E. K. H. Salje, H. Zhang, H. Idrissi, D. Schryvers, M. A. Carpenter, X. Moya, and A. Planes, Mechanical resonance of the austenite/martensite interface and the pinning of the martensitic microstructures by dislocations in $\mathrm{Cu}_{74.08} \mathrm{Al}_{23.13} \mathrm{Be}_{2.79}$, Phys. Rev. B 80, 134114 (2009).

[9] F. J. Pérez-Reche, M. Stipcich, E. Vives, L. Mañosa, A. Planes, and M. Morin, Kinetics of martensitic transitions in $\mathrm{Cu}-\mathrm{Al}-\mathrm{Mn}$ under thermal cycling: Analysis at multiple length scales, Phys. Rev. B 69, 064101 (2004).

[10] C. M. Otsuka and K. Wayman (eds.), Shape Memory Materials (Cambridge University Press, Cambridge, 1998).

[11] M. C. Gallardo, J. Jiménez, and J. del Cerro, Experimental device for measuring the influence of a uniaxial stress on specific heat: Application to the strontium titanate ferroelastic crystal, Rev. Sci. Instrum. 66, 5288 (1995).
[12] J. del Cerro, F. J. Romero, M. C. Gallardo, S. A. Hayward, and J. Jiménez, Latent heat measurement near a tricritical point: A study of the $\mathrm{KMnF}_{3}$ ferroelastic crystal, Thermochim. Acta 343, 89 (2000).

[13] A. Clauset, C. Shalizi, and M. Newman, Power-law distributions in empirical data, SIAM Rev. 51, 661 (2009).

[14] E. K. H. Salje, A. Planes, and E. Vives, Analysis of crackling noise using the maximum-likelihood method: Power-law mixing and exponential damping, Phys. Rev. E 96, 042122 (2017).

[15] A. Planes and E. Vives, Avalanche criticality in thermal-driven martensitic transitions: The asymmetry of the forward and reverse transitions in shape-memory materials, J. Phys. Condens. Matter 29, 334001 (2017).

[16] A. Planes, L. Mañosa, D. Ríos-Jara, and J. Ortín, Martensitic transformation of Cu-based shape-memory alloys: Elastic anisotropy and entropy change, Phys. Rev. B 45, 7633 (1992).

[17] L. Mañosa, A. Planes, J. Ortín, and B. Martínez, Entropy change of martensitic transformations in Cu-based shape-memory alloys, Phys. Rev. B 48, 3611 (1993).

[18] A. Planes and L. Mañosa, in Solid State Phys._Adv. Res. Appl., edited by H. Ehrenreich and F. B. T.-S. S. P. Spaepen (Academic Press, San Diego, 2001), pp. 159-267.

[19] J. Baró, J.-M. Martín-Olalla, F. J. Romero, M. C. Gallardo, E. K. H. Salje, E. Vives, and A. Planes, Avalanche correlations in the martensitic transition of a $\mathrm{Cu}-\mathrm{Zn}-\mathrm{Al}$ shape memory alloy: Analysis of acoustic emission and calorimetry, J. Phys. Condens. Matter 26, 125401 (2014).

[20] D. L. Beke, M. K. Bolgár, L. Z. Tóth, and L. Daróczi, On the asymmetry of the forward and reverse martensitic transformations in shape memory alloys, J. Alloys Compd. 741, 106 (2018).

[21] X. Balandraud, N. Barrera, P. Biscari, M. Grédiac, and G. Zanzotto, Strain intermittency in shape-memory alloys, Phys. Rev. B 91, 174111 (2015). 